\title{
Application of potential outcomes to an intentional weight loss latent variable problem
}

\author{
Gary L. Gadbury, Thidaporn Supapakorn, \\ Christopher S. Coffey, Scott W. Keith, and David B. Allison
}

Studies that explore the link between weight loss among obese individuals and mortality have met with mixed results. One possible explanation is that total weight loss may have contributions from weight loss that is intentional and weight loss that is unintentional. The latter may be due to some underlying condition that has a deleterious effect on subsequent mortality. Some studies have then focused on subjects who intend to lose weight. However, in a population there is no guarantee that weight loss among these individuals is due only to their intention. This paper extends the work of Coffey et al. (2005) who treated intentional weight loss as a latent variable. In particular, the problem is reformulated using potential outcomes. This formulation more clearly identifies a nonestimable correlation that arises because of the latent variable, and it allows for the incorporation of covariate information that can tighten estimable bounds for this correlation. We show in a data set from an experiment on mice that substantial tightening of bounds is possible with a covariate that is predictive of weight loss. These bounds can then, in turn, be used to estimate bounds on a causal parameter in a linear model.

Keywords And Phrases: Causality, Mortality, Nonestimable, Potential outcomes, Weight loss.

\section{INTRODUCTION}

Obesity is associated with increased mortality rate (Allison et al., 1999) and short-term weight loss improves risk factors for mortality (Weinsier, 1987); however, it has not been convincingly shown that weight loss among obese persons results in reduced mortality rate. Total weight loss for individuals in a population is a sum of intentional weight loss (IWL) and unintentional weight loss (UWL). Few studies are designed to measure the "intention to lose weight" (Sorensen, 2003), and those that do must often implicitly assume that subsequent observed weight loss was due to the intention to lose weight (Yang et al., 2003). Yet among subjects intending to lose weight, only total weight loss can be observed and this total, as well as any apparent effect on mortality could have contributions from UWL and IWL (Yang et al., 2003). These contributions may be in opposite

directions from one another, as might be the case if UWL resulted from some underlying disease in a subset of the population being studied.

Different definitions and terminology have been used when describing latent variables (Bollen, 2002). Herein we note that total weight loss among subjects intending to lose weight is observable, but IWL itself is not. Thus, IWL is the latent variable that is of interest. Coffey et al. (2005) developed a framework using IWL as a latent variable and showed that the effect of IWL on mortality is a nonestimable parameter (unless one is willing to make certain strong assumptions about other parameters). This paper reformulates Coffey et al. (2005) using the potential outcomes framework (Rubin, 1974). Potential outcomes help to clarify nonestimable quantities, and they are used herein to tighten bounds for a nonestimable correlation parameter and a causal parameter in a linear model. In general, the determination of causality is of course complex and detailed treatments of the topic as well as key axioms can be found in Shipley (2000).

Aside from Coffey et al. (2005) we know of no other papers that consider total weight loss as a sum of UWL and IWL and that specifically seek to quantify the effect of IWL on mortality or some other subsequent measure of health. There is a large body of literature on latent variable models with much of it focused on causality in observational data (cf., Berkane, 1997). Some such papers consider assignment to treatment as being dependent upon a latent variable in nonrandomized studies with resulting inferences then sensitive to hidden bias (e.g., Rosenbaum, 1991). Sensitivity analyses can be used to bound effects of treatment in such contexts (e.g., Rosenbaum, 1995; Heckman and Vytlach, 1999). Here the latent variable is the causal variable that is of interest and assignment to intention is assumed to be random as was the case in Coffey et al. (2005) and is, in fact, true in the mice data example presented later. Estimation of a direct effect on a response from a variable that is not observable poses obvious problems; however, aspects of the study design combined with the fact that IWL is partially observable in total weight loss yield enough information about the variable that bounds for its effect can be estimated.

In the next section, the key results of Coffey et al. (2005) are reviewed and the problem is re-formulated using potential outcomes. Section 3 considers the role of a covariate for 
tightening bounds for a causal parameter. Section 4 illustrates a method to tighten bounds using the data example from Coffey et al. (2005). A parametric bootstrap procedure is then used to investigate sampling variability of estimated bounds. We conclude with a discussion.

\section{INTENTIONAL VERSUS UNINTENTIONAL WEIGHT LOSS ON MORTALITY}

\subsection{A brief review of Coffey et al. (2005)}

Assume that subjects are observed until the time of death and denote the time until death (or some monotonic transformation thereof) as $Y$. More generally, the variable $Y$ can be any continuous measurement of health or wellness. Let $X$ be an indicator variable where $X=0$ for subjects not intending to lose weight and $X=1$ for subjects intending to lose weight. Denote the weight lost due to a subject's or experimenter's intention as $Z(Z=$ IWL) and the weight lost due to factors other than the intention as $W(W=\mathrm{UWL})$. Further, assume that $Z=0$ when $X=0$ so we have that $Z \mid X=1 \sim\left(\mu_{Z}, \sigma_{Z}^{2}\right)$ and $P(Z=0 \mid X=0)=1$.

Coffey et al. (2005) considered the following linear model,

$$
Y=\beta_{0}+\beta_{1} W+\beta_{2} Z X+\beta_{3} X+e,
$$

where $e$ is a random error term. Slope coefficients $\beta_{1}, \beta_{2}, \beta_{3}$ are defined herein as partial regression coefficients in a general linear model (Graybill, 1976) and, when estimable, are estimated via ordinary least squares.

The parameter $\beta_{1}$ captures the effect of UWL on $Y$ and $\beta_{2}$ captures the effect of IWL on $Y$. The model assumed an interaction between $Z$ and $X$, but it assumed no interaction between $W$ and $X$. The parameter $\beta_{3}$ allowed for the fact that there may be some effect of intending to lose weight (or more likely the actions or conditions that follow from such intention) as some data suggest (Gregg et al., 2003, 2004) that is associated with mortality. Levels of $X$ were assumed to be "assigned" at random so that if $Z$ could be observed for some subjects, unbiased estimates for $\beta_{2}$ in equation (1) could be obtained. This random assignment assumption is unlikely to hold in human studies and the implications due to violation of the assumption are left as discussion points later on.

In practice we are only able to observe total weight loss, $V=W+Z$ so when $X=0$, a regression model based on observable data is,

$$
Y=\beta_{0}+\beta_{1} W+e,
$$

and when $X=1$, a regression model (again based on observable data) is:

$$
Y=\lambda_{0}+\lambda_{1} V+e
$$

Note that (3) does not follow from (1) when $X=1$ because the variable $Z$ in (1) is not separately observable. Because of this, one might refer to (3) as a naïve model relating mortality to total weight loss. Coffey et al. (2005) showed that $\beta_{2}$ can be written as,

$$
\beta_{2}=\frac{\lambda_{1} \sigma_{V}^{2}-\beta_{1}\left(\sigma_{W}^{2}+\rho_{W, Z} \sigma_{W} \sigma_{Z}\right)}{\sigma_{Z}^{2}+\rho_{W, Z} \sigma_{W} \sigma_{Z}} .
$$

The parameters, $\beta_{1}, \lambda_{1}, \sigma_{V}^{2}$ and $\sigma_{W}^{2}$ are estimable in observed data, but the parameters $\rho_{W, Z}$ (the simple correlation between $W$ and $Z$ ) and $\sigma_{Z}$ are not since $Z$ is not observable. Coffey et al. (2005) used (4) to assess the sensitivity of $\beta_{2}$ to varying $\rho_{W, Z}$ and $\sigma_{Z}$, plugging in sample estimates for the other parameters $\beta_{1}, \lambda_{1}, \sigma_{V}^{2}$ and $\sigma_{W}^{2}$.

\subsection{Formulating the IWL problem with potential outcomes-no covariate}

The potential outcomes for the IWL problem can be defined as, $\left(Y^{(x)}, W^{(x)}, Z\right)$, where $W, Z$ are as before and $x=0$ for subjects in the unintentional group and $x=1$ for subjects in the intentional weight loss group. There are five potential outcomes for a subject where, for example, $Y^{(0)}, Y^{(1)}$ are the mortality of a subject not intending to lose weight and intending to lose weight, respectively. Similarly, $W^{(0)}, W^{(1)}$ are the unintentional weight loss for a subject that does not and does, respectively, intend to lose weight. As in Coffey et al. (2005), the two variables $W^{(0)}, W^{(1)}$ are assumed to have the same variance, $\sigma_{W}^{2}$. Since it was assumed that $Z^{(0)}=0$, we omit the superscript for $Z$ and treat $Z^{(1)}=Z$ as a single outcome, though unobservable, for an individual. Models of interest that were considered in Coffey et al. (2005) in terms of potential outcomes are,

$$
\begin{aligned}
& Y^{(0)}=\beta_{0}+\beta_{1} W^{(0)}+\varepsilon^{(0)}, \\
& Y^{(1)}=\left(\beta_{0}+\beta_{3}\right)+\beta_{1} W^{(1)}+\beta_{2} Z+\varepsilon^{(1)},
\end{aligned}
$$

where the two random error terms are assumed to have mean zero. The causal but nonestimable causal effect of IWL is

$D\left(W^{(x)}, Z\right)=E\left(Y^{(1)}-Y^{(0)}\right)=\beta_{3}+\beta_{1}\left(W^{(1)}-W^{(0)}\right)+\beta_{2} Z$,

where the expectation is conditional on the weight loss variables. For a 1 unit change in $Z$, holding other variables constant, the effect is $D(z+1)-D(z)=\beta_{2}$. The parameter $\beta_{2}$ will be called the causal parameter and equation (6), the causal model.

For a given subject, either $\left(Y^{(0)}, W^{(0)}\right)$ or $\left(Y^{(1)}, V^{(1)}=\right.$ $W^{(1)}+Z$ ) are observed depending on assignment to the unintentional or intentional group. Since $V^{(0)}=W^{(0)}$, we drop the superscript for $V$ and let $V=W^{(1)}+Z$. The two resulting regression equations given by equations (2) and (3) provide estimates of $\beta_{1}$ and $\lambda_{1}$. Consider a third simple linear regression model,

$$
W^{(1)}=\alpha_{0}+\alpha_{1} V+\varepsilon .
$$


Table 1. Parameter Notation, Descriptions of Parameters Including Restrictions on Marginal and Joint Distributions, and Parameter Estimates from the Data Example

\begin{tabular}{|c|c|c|}
\hline Parameter notation & Parameter description & Parameter estimate \\
\hline$\mu_{Y^{(1)}}$ & $E\left(Y^{(1)}\right)$ & 36.91 \\
\hline$\mu_{Y^{(0)}}$ & $E\left(Y^{(0)}\right)$ & 33.71 \\
\hline$\mu_{W}$ & $E\left(W^{(1)}\right)=E\left(W^{(0)}\right)$ & 0.45 \\
\hline$\mu_{V}$ & $E\left(V^{(1)}\right)=E(V), V=W+Z$ & 14.14 \\
\hline$\mu_{U}$ & $E(U)$ & 41.72 \\
\hline$\sigma_{Y^{(1)}}^{2}$ & $\operatorname{Var}\left(Y^{(1)}\right)$ & 5.42 \\
\hline$\sigma_{Y^{(0)}}^{2}$ & $\operatorname{Var}\left(Y^{(0)}\right)$ & 4.71 \\
\hline$\sigma_{W}^{2}$ & $\operatorname{Var}\left(W^{(1)}\right)=\operatorname{Var}\left(W^{(0)}\right)$ & 4.79 \\
\hline$\sigma_{V}^{2}$ & $\operatorname{Var}\left(V^{(1)}\right)=\operatorname{Var}(V)$ & 5.20 \\
\hline$\sigma_{U}^{2}$ & $\operatorname{Var}(U)$ & 4.68 \\
\hline$\rho_{Y^{(1)}, V}$ & $\operatorname{Cor}\left(Y^{(1)}, V^{(1)}\right)=\operatorname{Cor}\left(Y^{(1)}, V\right)$ & 0.105 \\
\hline$\rho_{Y^{(0)}, W}$ & $\operatorname{Cor}\left(Y^{(0)}, W(0)\right.$ & -0.436 \\
\hline$\rho_{Y^{(1)}, U}$ & $\operatorname{Cor}\left(Y^{(1)}, U\right)$ & 0.126 \\
\hline$\rho_{Y^{(0)}, U}$ & $\operatorname{Cor}\left(Y^{(0)}, U\right)$ & -0.272 \\
\hline$\rho_{W, U}$ & $\operatorname{Cor}\left(W^{(1)}, U\right)=\operatorname{Cor}\left(W^{(0)}, U\right)$ & 0.550 \\
\hline$\rho_{V, U}$ & $\operatorname{Cor}\left(V^{(1)}, U\right)=\operatorname{Cor}(\operatorname{V}, U)$ & 0.870 \\
\hline & & \\
\hline
\end{tabular}

The parameters in (7) cannot be estimated since the pair, $\left(W^{(1)}, V\right)$ cannot be separately observed on the same subject. It can be shown that, $\beta_{2}=\frac{\lambda_{1}-\beta_{1} \alpha_{1}}{1-\alpha_{1}}$ which is a function of one nonestimable parameter, $\alpha_{1}$. Assuming $W^{(1)}, V$ independent implies that $\alpha_{1}=0$ and that $\beta_{2}=\lambda_{1}$, an assumption noted in Coffey et al. (2005) that is unlikely to be true. We call the estimate of $\lambda_{1}$ in the regression model (3) a naïve estimate of $\beta_{2}$. It is a nonestimable correlation between $V$ and $W^{(1)}$ that makes $\alpha_{1}$ not estimable in (7). The result in (4) that was a function of two nonestimable parameters can be rewritten as a function of only one nonestimable correlation, $\rho_{W, V}$. To simplify notation, we drop the superscripts on the variables in the correlation because $\rho_{W, V}$, for example, will imply a correlation between $V$ and $W^{(1)}$, since $V^{(0)}=W^{(0)}$. For reference, we summarized the notation for distributional parameters of potential outcomes variables that are estimable along with their description and any assumed restrictions on marginal and joint distributions in Table 1. Table 1 also includes information on a covariate $U$ (to be described later), and parameter estimates from a data example to be described in Section 4.

Write the causal model in (6) as,

$$
\begin{aligned}
Y^{(1)} & =\left(\beta_{0}+\beta_{3}\right)+\beta_{1} W^{(1)}+\beta_{2}\left(V-W^{(1)}\right)+\varepsilon^{(1)} \\
& =\left(\beta_{0}+\beta_{3}\right)+\left(\beta_{1}-\beta_{2}\right) W^{(1)}+\beta_{2} V+\varepsilon^{(1)} .
\end{aligned}
$$

In the causal model (8) above, $W^{(1)}$ is not observable. However, $\left(Y^{(0)}, W^{(0)}\right)$ are observable in (5) for subjects not intending to lose weight and we have assumed that the parameter $\beta_{1}$ is the same in models (5) and (8) (i.e., it was assumed that there was no $W$ by $X$ interaction in model (1)). Equation (4), using the identities, $\rho_{W^{(1)}, Z} \sigma_{Z}=\sigma_{V} \rho_{W, V}-\sigma_{W}$ and $\sigma_{Z}^{2}=\sigma_{V}^{2}+\sigma_{W}^{2}-2 \sigma_{V} \sigma_{W} \rho_{W, V}$, results in the following:

$$
\begin{aligned}
\beta_{2}= & \frac{\lambda_{1} \sigma_{V}^{2}-\beta_{1}\left[\sigma_{W}^{2}+\sigma_{W}\left(\sigma_{V} \rho_{W, V}-\sigma_{W}\right)\right]}{\sigma_{Z}^{2}+\sigma_{W}\left(\sigma_{V} \rho_{W, V}-\sigma_{W}\right)} \\
= & \frac{\lambda_{1} \sigma_{V}^{2}-\beta_{1} \sigma_{W}^{2}-\beta_{1} \sigma_{W} \sigma_{V} \rho_{W, V}+\beta_{1} \sigma_{W}^{2}}{\sigma_{W}^{2}+\sigma_{V}^{2}-2 \sigma_{W} \sigma_{V} \rho_{W, V}+\sigma_{W} \sigma_{V} \rho_{W, V}-\sigma_{W}^{2}} \\
= & \frac{\lambda_{1} \sigma_{V}^{2}-\beta_{1} \sigma_{W} \sigma_{V} \rho_{W, V}}{\sigma_{V}^{2}-2 \sigma_{W} \sigma_{V} \rho_{W, V}+\sigma_{W} \sigma_{V} \rho_{W, V}} \\
= & \frac{\sigma_{Y^{(1)}, V}-\frac{\sigma_{Y(0)}, W}{\sigma_{W}^{2}} \sigma_{W} \sigma_{V} \rho_{W, V}}{\sigma_{V}\left(\sigma_{V}-\sigma_{W} \rho_{W, V}\right)} \\
= & \frac{\sigma_{Y^{(1)}} \sigma_{V} \rho_{Y^{(1)}, V}-\frac{\sigma_{Y}(0)}{\sigma_{W} \rho_{Y(0)}}}{\sigma_{W}^{2}} \sigma_{W} \sigma_{V} \rho_{W, V} \\
= & \frac{\sigma_{Y^{(1)}} \rho_{Y^{(1)}, V}-\sigma_{Y}-\sigma_{W} \rho_{Y(0), W} \rho_{W, V}}{\sigma_{V}-\sigma_{W} \rho_{W, V}} .
\end{aligned}
$$

The two correlations, $\rho_{Y^{(1)}, V}, \rho_{Y^{(0)}, W}$ in equation (9) are estimable using observable data as are the four standard deviations. Letting the nonestimable correlation $\rho_{W, V}$ go from -1 to 1 provides bounds for $\beta_{2}$ that can be estimated from observed data.

There is another constraint that tightens the bounds for $\beta_{2}$. Observe that the random vector, $\left(Y^{(0)}, Y^{(1)}, W^{(1)}\right.$, $V)^{\prime}$, has a correlation matrix with six correlation parameters, four of which are nonestimable, $\rho_{Y^{(1)}, Y^{(0)}}, \rho_{Y^{(1)}, W^{(1)}}$, $\rho_{Y^{(0)}, V}, \rho_{W, V}$. Gadbury and Iyer (2000) used the positive definiteness requirement for a correlation matrix to produce a set of plausible values for a nonestimable correlation. Applying this idea here, however, is not helpful since the positive definiteness requirements result in 3 equations 
with 4 nonestimable parameters. Consider the distribution of $\left(Y^{(1)}, W^{(1)}, V\right)^{\prime}$ and observe that these 3 variables are, in fact, those if interest in the causal model given by equation (8). Let $R$ be the 3-dimensional correlation matrix and note that there are 2 nonestimable parameters in $R, \rho_{Y^{(1)}, W}$ and $\rho_{W, V}$, but they are functionally related under the assumptions described herein. Equation (8) combined with equation (5), which gives a formula for $\beta_{1}$ produces two formulas for $\beta_{2}$. Equating these and solving leads to another derivation for $\beta_{2}$ that was given in equation (9). Some details of this are given in Appendix A. One result is a relationship between the two nonestimable parameters given by,

$\rho_{Y^{(1)}, W^{(1)}}$

$=\frac{\sigma_{Y^{(0)}} \sigma_{V} \rho_{Y^{(0)}, W}\left(1-\rho_{W, V}^{2}\right)+\sigma_{Y^{(1)}} \rho_{Y^{(1)}, V}\left(\sigma_{V} \rho_{W, V}-\sigma_{W}\right)}{\sigma_{Y^{(1)}}\left(\sigma_{V}-\sigma_{W} \rho_{W, V}\right)}$.

Plugging in point estimates for estimable quantities in (10), gives a correlation matrix for $\left(Y^{(1)}, W^{(1)}, V\right)^{\prime}$ of the form,

$$
R=\left(\begin{array}{ccc}
1 & h\left(\rho_{W, V}\right) & \rho_{Y^{(1)}, V} \\
h\left(\rho_{W, V}\right) & 1 & \rho_{W, V} \\
\rho_{Y^{(1)}, V} & \rho_{W, V} & 1
\end{array}\right)
$$

where $h\left(\rho_{W, V}\right)$ is given by the right hand side of equation (10). Let $e_{\min }$ be the minimum eigenvalue of the correlation matrix, $R$. The constraint, $e_{\min }>0$ can tighten the range of plausible values of $\rho_{W, V}$. The range for $\rho_{W, V}$ results in bounds for $\beta_{2}$ that can be estimated from observable data.

\section{THE ROLE OF A COVARIATE IN TIGHTENING BOUNDS}

Suppose that a covariate, $U$, is observed on all subjects in a study and that $U$ is not affected by the assigned treatment (i.e., intention). An example of such a covariate is baseline weight at the beginning of the period of intention to lose or not to lose weight. A question arises about whether the causal model is given by equation (8) that was defined without the covariate, or by a similar model that includes the covariate. The ability of the covariate to substantially tighten the bounds for $\rho_{W, V}$ depends on its ability to predict $W$ and/or $V$, in which case collinearity problems are encountered when it is included in the causal model. Which causal model is ultimately preferred depends on the ability of $U$ to predict $Y^{(1)}$ given that $W$ and $V$ are in the model. We present a method to estimate bounds for $\beta_{2}$ in a causal model both with and without a covariate in it. The issue of collinearity is discussed further via a data example in Section 4 .

\subsection{Using a covariate to tighten the range for $\rho_{W, V}$}

The 4-dimensional correlation matrix for the random vector $\left(Y^{(1)}, W^{(1)}, V, U\right)^{\prime}$ is given by,
(12) $\Sigma=\left(\begin{array}{cccc}1 & \rho_{Y^{(1)}, W^{(1)}} & \rho_{Y^{(1)}, V} & \rho_{Y^{(1)}, U} \\ \rho_{Y^{(1)}, W^{(1)}} & 1 & \rho_{W, V} & \rho_{W, U} \\ \rho_{Y^{(1)}, V} & \rho_{W, V} & 1 & \rho_{V, U} \\ \rho_{Y^{(1)}, U} & \rho_{W, U} & \rho_{V, U} & 1\end{array}\right)$

Of the 6 correlation parameters in (12), all are estimable except the same two from the previous section, $\rho_{Y^{(1)}, W^{(1)}}$ and $\rho_{W, V}$, since the covariate $U$ is observable for all subjects. Partition the correlation matrix as,

$$
\Sigma=\left(\begin{array}{cc}
R & S_{12} \\
S_{21} & S_{22}
\end{array}\right)
$$

where $R$ is the same matrix given by (11) where the relationship $\rho_{Y^{(1)}, W^{(1)}}=h\left(\rho_{W, V}\right)$ was established using the multivariate distribution without the covariate. Thus $\rho_{W, V}$ has already been constrained to values making $R$ positive definite, i.e., $R>0$. The notation, $S_{12}=S_{21}^{\prime}$ is used for the possible more general case where there are multiple covariates observed for each subject and these terms represent the correlation between each covariate and the 3 variables, $\left(Y^{(1)}, W^{(1)}, V\right)$. The matrix $S_{22}$ is the correlation matrix of the vector of covariates, and it is equal to 1 in the case of only one covariate. The constraint that $R>0$ combined with the identity, $|\Sigma|=|R|\left|S_{22}-S_{21} R^{-1} S_{12}\right|>0$ provides the information to further constrain $\rho_{W, V}$. With only one covariate, this simplifies to $S_{21} R^{-1} S_{12}<1$.

\subsection{A causal model with a covariate}

The full model with a covariate considered here is,

(14) $Y=\beta_{0}+\beta_{1} W+\beta_{2} Z X+\beta_{3} U+\beta_{4} X+\beta_{5} U X+\varepsilon$,

resulting in the two models for the UWL and IWL groups given by equations (15) and (16), respectively.

(15) $Y^{(0)}=\beta_{0}+\beta_{1} W^{(0)}+\beta_{3} U+\varepsilon^{(0)}$,

$$
\begin{aligned}
Y^{(1)}= & \left(\beta_{0}+\beta_{4}\right)+\beta_{1} W^{(1)}+\beta_{2} Z+\left(\beta_{3}+\beta_{5}\right) U+\varepsilon^{(1)} \\
= & \left(\beta_{0}+\beta_{4}\right)+\left(\beta_{1}-\beta_{2}\right) W^{(1)}+\beta_{2} V \\
& +\left(\beta_{3}+\beta_{5}\right) U+\varepsilon^{(1)} .
\end{aligned}
$$

The treatment-covariate interaction term in equation (14) implies that the slope parameters on the covariate, $U$, are not necessarily equal in the two groups. The causal parameter is $\beta_{2}$ in the causal model given by equation (16) because, $D\left(Z, W^{(x)}, U\right)=E\left(Y^{(1)}-Y^{(0)}\right)=\beta_{4}+\beta_{1}\left(W^{(1)}-W^{(0)}\right)+$ $\beta_{2} Z+\beta_{5} U$ and, for a one unit change in $Z$, the corresponding change in $E\left(Y^{(1)}-Y^{(0)}\right)$ is $\beta_{2}$, holding other variables fixed.

Derivation of results using this model is similar to that shown in Appendix A for the case with no covariate. Equation (15) gives $\beta_{1}=\frac{\operatorname{Cov}\left(Y^{(0)}, W^{(0)} \mid U\right)}{\operatorname{Var}\left(W^{(0)} \mid U\right)}$ and equation (16) combined with (15) give two forms for $\beta_{2}, \beta_{2}^{*}=$ 
$\frac{\operatorname{Cov}\left(Y^{(1)}, V \mid W^{(1)}, U\right)}{\operatorname{Var}\left(V \mid W^{(1)}, U\right)}$ and $\beta_{2}^{* *}=\beta_{1}-\frac{\operatorname{Cov}\left(Y^{(1)}, W^{(1)} \mid V, U\right)}{\operatorname{Var}\left(W^{(1)} \mid V, U\right)}$. Equating the two forms results in a functional relationship, $\rho_{Y^{(1)}, W^{(1)}}=g\left(\rho_{W, V}\right)$. The result is an equation for $\beta_{2}$ that is a function of only one nonestimable correlation, $\rho_{W, V}$. The derivation for $\beta_{2}$, though straightforward, is somewhat tedious with some steps given in Appendix B. The constraints on $\rho_{W, V}$ result from the positive definiteness requirement for $\Sigma$ in (12), and these constraints can be used to bound $\beta_{2}$ in the model given by (16).

\subsection{Putting it all together using a covariate}

The nonestimable correlation, $\rho_{W, V}$, is an unknown, but fixed parameter. The following shows that $\rho_{W, V}$ is bounded by a continuous interval under certain conditions.

Proposition 1. Consider a population correlation matrix consisting of an unknown nonestimable correlation, $\rho_{W, V}$, and other distinct but fixed known correlations. Assume that there is at least one value of $\rho_{W, V}$ such that the population correlation matrix is positive definite. Then plausible values of $\rho_{W, V}$ are bounded by a continuous interval.

Proof. Let a random vector be given by $(V, W, U)$ where $U$ is a vector of variables. Partition the correlation matrix as $\left(\begin{array}{cc}A & B \\ B^{\prime} & S_{U}\end{array}\right)$ where $A=\left(\begin{array}{cc}1 & \rho_{W V} \\ \rho_{W V} & 1\end{array}\right), S_{U}$ is a correlation matrix of $U$, and $B=\left(\begin{array}{c}S_{V U} \\ S_{W U}\end{array}\right)$, i.e., the correlations of $V$ and $W$, respectively, with variables in $U$. The positive definiteness of a correlation matrix implies that $\left|A-B S_{U}^{-1} B^{\prime}\right|>0$. Write $B S_{U}^{-1} B^{\prime}=\left(\begin{array}{ll}m_{11} & m_{12} \\ m_{21} & m_{22}\end{array}\right)$ where $m_{12}=m_{21}$. Then $\rho_{W, V}$ is bounded by $m_{12} \pm \sqrt{\left(1-m_{11}\right)\left(1-m_{22}\right)}$.

The proposition is important to state because it is difficult to interpret bounds for $\rho_{W, V}$ when they do not contain a continuous interval. In the application described here, the conditions of the proposition are not met because of a restriction imposed by the assumption that the coefficient $\beta_{1}$ was the same in both IWL and UWL groups. This assumption helped to tighten bounds for $\rho_{W, V}$ but also led to the two parameters, $\rho_{W, V}$ and $\rho_{Y(1)}, W$, being functionally related by either equation (10) as used to bound $\rho_{W, V}$ in Section 3.1, or by equation (B1) in Appendix B, as used to bound $\rho_{W, V}$ in Section 3.2. As a result, plausible values of $\rho_{W, V}$ may not lie in a continuous interval, but this is an artifact of an assumption made herein. So we seek a reasonable technique to combine information obtained from population models with and without a covariate that produces a continuous set of plausible values. Let $\mathrm{P}_{1}$ be the estimated set of plausible values for $\rho_{W, V}$ using Section 3.1, and $\mathrm{P}_{2}$ be the estimated set of plausible values using Section 3.2. Since $\rho_{W, V}$ is a fixed population parameter, all of the information available in both the marginal trivariate distribution of $\left(Y^{(1)}, W^{(1)}, V\right)^{\prime}$ and in the 4-dimensional distribution of $\left(Y^{(1)}, W^{(1)}, V, U\right)^{\prime}$ is used. Thus we propose using values of $\rho_{W, V}$ such that

$$
\rho_{W, V} \in \mathrm{P}=\left(\mathrm{P}_{1} \cap \mathrm{P}_{2}\right)
$$

Once the set $\mathrm{P}$ has been obtained as in (17), bounds for $\beta_{2}$ can be presented using either equation (9) and/or equation (B2) in Appendix B, depending on whether one wants the estimated causal effect conditioned on the covariate. Hereafter we refer to estimated bounds as "valid bounds" when the lower and upper values bound a continuous interval.

\section{ILLUSTRATION ON A DATA SET}

We illustrate our approach with an example described in Coffey et al. (2005). The data were from 135 male mice of the $\mathrm{B} 10 \mathrm{C} 3 \mathrm{~F}_{1}$ strain that were fed ad libitum until 12 months of age at which point they were randomized, individually housed, and provided an intake of either an amount sufficient to maintain body weight (a control diet is the unintentional condition, $X=0,160 \mathrm{kcal} /$ mouse $/ \mathrm{wk}$ ) or an intake of $90 \mathrm{kcal}$ (the intentional condition $X=1$ ). This laboratory experiment allowed that animals be followed until all are dead and the assignment to condition to be random.

At 23 months the weight loss was observed for each mouse. The 12 month baseline weight is a covariate, $U$. Four mice were not alive at the 23 month weight recording and were omitted. The remaining 131 mice were followed until death at which their lifespan, $Y=$ mortality, was recorded for 67 mice in the IWL group and 64 in the UWL group.

\subsection{Analysis without the covariate}

Figure 1 shows the plot of pairs $\left(V, Y^{(1)}\right)$ and $\left(W^{(0)}, Y^{(0)}\right)$ with a fitted simple linear regression line to each group.

The IWL group tended to have more weight loss and a slight positive relationship with mortality. The UWL group suggests a negative association between $W$ and $Y^{(0)}$. Sample estimates, given in Table 1, were computed for all estimable parameters. The correlation matrix, $R$ (defined in equation (11)), is positive definite for values of $\rho_{W, V}$ in each of the two intervals, $(-1,0.730)$ and $(0.980,1)$, but is near singularity for $\rho_{W, V}$ in $(0.980,1)$. The ranges of plausible values for $\rho_{W, V}$ were obtained numerically such that the minimum eigenvalue $e_{\min }$ of the matrix $R$ is positive. The discontinuous interval is due to nonlinearity in the functional relationship, $\rho_{Y^{(1)}, W^{(1)}}=h\left(\rho_{W, V}\right)$ given by equation (10), creating a higher order functional relationship between $e_{\min }$ and $\rho_{W, V}$ than would otherwise be the case if $\rho_{Y^{(1)}, W^{(1)}}$ and $\rho_{W, V}$ were distinct parameters. The estimated plausible values for $\beta_{2}$ based on the allowable values for $\rho_{W, V}$ are $-0.15 \leq \beta_{2} \leq 1.21$, and $5.11 \leq \beta_{2} \leq 6.41$. Coffey et al. (2005) excluded the upper range as implausible based on knowledge of the particular application, but there was no mathematical justification for eliminating this range. ${ }^{1}$ Unless one is willing to assume a more restricted range for $\rho_{W, V}$, there is little else that can be done to tighten the bounds for $\beta_{2}$ without more information.

\footnotetext{
${ }^{1}$ The results reported here for the data example are based on weight loss measured in original units of grams. The example in Coffey et al. (2005) used weight loss in 5 gram increments. Thus bounds for $\beta_{2}$ in Coffey et al., are 5 times those reported here.
} 


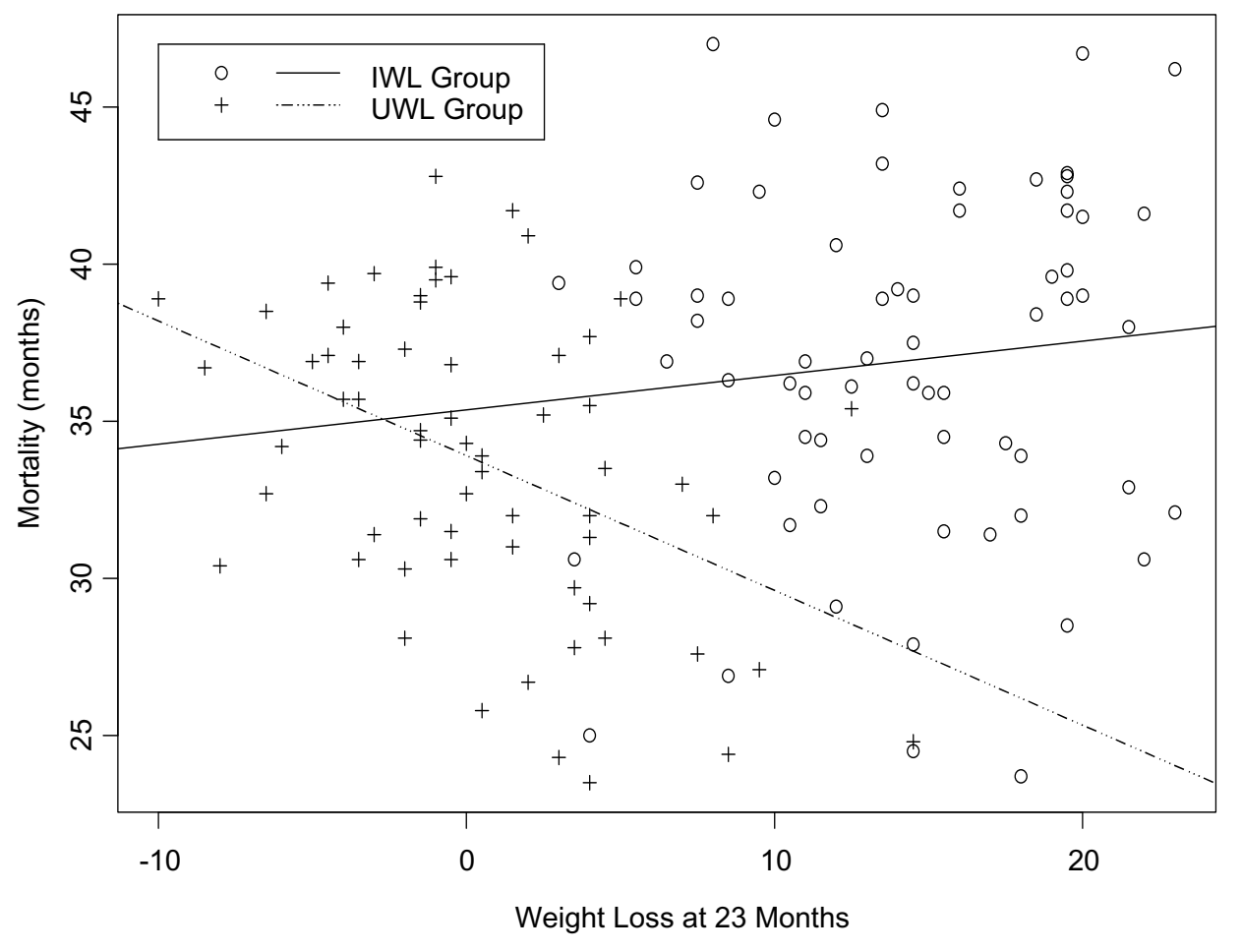

Figure 1. Plot of Mortality Versus Observed Weight Loss with Fitted Regression Lines for the Two Groups.

\subsection{Illustration on the data set using baseline weight as a covariate}

The covariate $U$ is baseline weight, in grams, that was recorded prior to assignment to the treatment or control group. The positive definiteness requirement for the 3dimensional correlation matrix for $\left(W^{(1)}, V, U\right)$ provides estimated bounds for the nonestimable correlation, $0.067 \leq$ $\rho_{\mathrm{W}, \mathrm{V}} \leq 0.890$. Using the 4-dimensional correlation matrix for $\left(Y^{(1)}, W^{(1)}, V, U\right), \Sigma$ as defined in equation (13) provides estimated bounds, $0.203 \leq \rho_{\mathrm{W}, \mathrm{V}} \leq 0.720$.

Redefining the relationship between $\rho_{Y^{(1)}, W^{(1)}}$ and $\rho_{W, V}$ as outlined in Section 3.2 (using equation (B1) in Appendix B), the restriction that $\Sigma$ in equation (12) be positive definite results in a set of plausible values for $\rho_{W, V}$ that is the union of the two intervals, $(0.069,0.676)$ and $(0.792$, $0.890)$. The intervals are not connected due to the nonlinear relationship $\rho_{Y^{(1)}, W^{(1)}}=g\left(\rho_{W, V}\right)$ described in Section 3.2 and given in appendix equation (B1).

As discussed in Section 3.3, there are two estimated sets of plausible values for $\rho_{W, V}, \mathrm{P}_{1}=(0.203,0.720)$ and $\mathrm{P}_{2}=$ $[(0.069,0.676) \cup(0.792,0.890)]$. As proposed in Section 3.3, the intersection of these two sets produces estimated bounds $0.203 \leq \rho_{\mathrm{W}, \mathrm{V}} \leq 0.676$.

Using this interval as a range for values of $\rho_{W, V}$, we obtain bounds for the causal parameter $\beta_{2}$. Let $\beta_{2}^{\text {No }}$ be the parameter $\beta_{2}$ in the model without the covariate, i.e., equation (9). The estimated bounds are given by $0.233 \leq \beta_{2}^{N o} \leq$ 0.996. The naïve estimate of $\beta_{2}^{N o}$, i.e., $\lambda_{1}$ in equation (3), is 0.109 , which is below the estimated minimum for $\beta_{2}^{N o}$. This suggests that the positive effects of $Z$ on mortality are greater than what is observed to be the effect of total weight loss on mortality.

If $\beta_{2}^{Y e s}$ denotes $\beta_{2}$ in the model with the covariate as described in Section 3.2 and using equation (B2), the estimated bounds are given by $-0.216 \leq \beta_{2}^{\text {Yes }} \leq 1.125$. The naïve estimate of total weight loss on mortality in a model that includes the covariate is equal to -0.020 which is near the bottom range for the estimates of $\beta_{2}^{Y e s}$. The wider estimated range in the model that includes the covariate may be, in part, because baseline weight for this example is not statistically significant when included in the two regression models given by equations (2) and (3). Thus additional correlation parameters are being estimated for a variable that is not a significant predictor of mortality, given that the weight loss variable is in the model. Baseline weight is a significant predictor of $W$ and $V$, which explains its usefulness in tightening the range of plausible values for $\rho_{W, V}$, but it presents collinearity problems when included in the causal model. More discussion of this is in Section 5.

\subsection{Assessing the sampling variability of the bounds using a modified parametric bootstrap procedure}

A parametric bootstrap (Efron and Tibshirani, 1993) procedure was used to assess the sampling variability of the estimated bounds for $\rho_{W, V}, \beta_{2}^{N o}$, and $\beta_{2}^{\text {Yes }}$. A multivariate 
Table 2. The Results for Estimated Bounds for $\rho_{W, V}$ from 200 Parametric Bootstrap Simulations. $N$ Is the Total Sample Size Divided into Two Groups of Size N/2, $n_{p}$ Is the Number of the Sample Matrices out of 200 Simulations which Are Positive Definite, $n_{c}$ Is the Number of Simulations that Yield a Continuous Interval Between the Estimated Bounds, and Mean and sd Are the Mean and Standard Deviations of the $n_{c}$ Estimated Valid Bounds

\begin{tabular}{|c|c|c|c|c|c|c|c|c|c|}
\hline \multirow{2}{*}{\multicolumn{2}{|c|}{$\begin{array}{r} \\
\rho_{W, V}\end{array}$}} & \multicolumn{2}{|c|}{100} & \multicolumn{2}{|c|}{500} & \multicolumn{2}{|c|}{1000} & \multicolumn{2}{|c|}{10000} \\
\hline & & & $\max$ & $\min$ & $\max$ & $\min$ & $\max$ & $\min$ & $\max$ \\
\hline \multirow{3}{*}{0.3} & mean & 0.215 & 0.606 & 0.214 & 0.630 & 0.220 & 0.647 & 0.204 & 0.674 \\
\hline & $\mathrm{sd}$ & 0.182 & 0.127 & 0.091 & 0.061 & 0.069 & 0.054 & 0.027 & 0.014 \\
\hline & $\left(n_{p}, n_{c}\right)$ & \multicolumn{2}{|c|}{$(148,72)$} & \multicolumn{2}{|c|}{$(169,114)$} & \multicolumn{2}{|c|}{$(190,147)$} & \multicolumn{2}{|c|}{$(200,200)$} \\
\hline \multirow{3}{*}{0.4} & mean & 0.200 & 0.620 & 0.218 & 0.640 & 0.220 & 0.645 & 0.205 & 0.674 \\
\hline & $\mathrm{sd}$ & 0.207 & 0.127 & 0.083 & 0.066 & 0.070 & 0.054 & 0.023 & 0.011 \\
\hline & $\left(n_{p}, n_{c}\right)$ & \multicolumn{2}{|c|}{$(151,66)$} & \multicolumn{2}{|c|}{$(175,120)$} & \multicolumn{2}{|c|}{$(191,153)$} & \multicolumn{2}{|c|}{$(200,200)$} \\
\hline \multirow{3}{*}{0.5} & mean & 0.170 & 0.606 & 0.225 & 0.645 & 0.224 & 0.647 & 0.206 & 0.674 \\
\hline & $\mathrm{sd}$ & 0.186 & 0.127 & 0.097 & 0.059 & 0.080 & 0.059 & 0.024 & 0.012 \\
\hline & $\left(n_{p}, n_{c}\right)$ & \multicolumn{2}{|c|}{$(139,68)$} & \multicolumn{2}{|c|}{$(174,128)$} & \multicolumn{2}{|c|}{$(186,144)$} & \multicolumn{2}{|c|}{$(200,199)$} \\
\hline \multirow{3}{*}{0.6} & mean & 0.148 & 0.592 & 0.221 & 0.634 & 0.221 & 0.651 & 0.204 & 0.675 \\
\hline & $\mathrm{sd}$ & 0.142 & 0.117 & 0.102 & 0.069 & 0.078 & 0.047 & 0.023 & 0.011 \\
\hline & $\left(n_{p}, n_{c}\right)$ & \multicolumn{2}{|c|}{$(141,68)$} & \multicolumn{2}{|c|}{$(178,126)$} & \multicolumn{2}{|c|}{$(188,148)$} & \multicolumn{2}{|c|}{$(200,200)$} \\
\hline
\end{tabular}

Table 3. Similar to Table 2 Except for the Parameter $\beta_{2}^{\text {No }}$. Shown Are the Mean and Standard Deviations of the $n_{c}$ Bounds that Contained a Continuous Interval for $\beta_{2}^{\text {No }}$

\begin{tabular}{|l|l|l|cc|cc|cc|cc|}
\hline \multicolumn{2}{|c|}{$N$} & \multicolumn{2}{c|}{100} & \multicolumn{2}{c|}{500} & \multicolumn{2}{c|}{1000} & \multicolumn{2}{c|}{10000} \\
\hline$\rho_{W, V}$ & $\beta_{2}^{N o}$ & & $\min$ & $\max$ & $\min$ & $\max$ & $\min$ & $\max$ & $\min$ & $\max$ \\
\hline \multirow{2}{*}{0.3} & \multirow{2}{*}{0.315} & mean & 0.206 & 0.814 & 0.272 & 0.919 & 0.262 & 0.946 & 0.235 & 0.993 \\
\cline { 3 - 11 } & & sd & 0.393 & 0.420 & 0.121 & 0.148 & 0.088 & 0.123 & 0.034 & 0.054 \\
\hline \multirow{2}{*}{0.4} & \multirow{2}{*}{0.423} & mean & 0.189 & 0.836 & 0.263 & 0.924 & 0.262 & 0.945 & 0.234 & 0.989 \\
\cline { 3 - 11 } & & sd & 0.411 & 0.509 & 0.101 & 0.153 & 0.087 & 0.114 & 0.030 & 0.051 \\
\hline \multirow{2}{*}{0.5} & \multirow{2}{*}{0.569} & mean & 0.182 & 0.807 & 0.280 & 0.963 & 0.263 & 0.938 & 0.237 & 0.989 \\
\cline { 3 - 10 } & & sd & 0.429 & 0.516 & 0.115 & 0.149 & 0.102 & 0.124 & 0.032 & 0.053 \\
\hline \multirow{2}{*}{0.6} & \multirow{2}{*}{0.774} & mean & 0.192 & 0.789 & 0.275 & 0.918 & 0.265 & 0.951 & 0.233 & 0.989 \\
\cline { 3 - 10 } & & sd & 0.161 & 0.275 & 0.122 & 0.168 & 0.097 & 0.117 & 0.029 & 0.047 \\
\hline
\end{tabular}

normal distribution was used to simulate data according to the two models in equations (15) and (16). Equation (15) corresponds to data from a random vector $\left(Y^{(0)}, W^{(0)}, U\right)^{\prime}$ and equation (16) from a random vector $\left(Y^{(1)}, W^{(1)}, V, U\right)^{\prime}$. Sample estimates from the mice data set were used as parameter values in the distribution and the nonestimable $\rho_{W, V}$ was set to values within the range specified by the estimated bounds, in particular 0.3, 0.4, 0.5, and 0.6. Values of $\rho_{W, V}$ outside the range specified by the estimated bounds would not be allowed in the simulation model because the correlation matrix would not be positive definite. The estimable parameters and their estimates that were used are given in Table 1.

For each value of $\rho_{W, V}, \rho_{Y^{(1)}, W^{(1)}}$ is computed using equation (B1) in Appendix B. The sample sizes that were considered were $N=100,500,1000$, and 10000 , where the $N$ samples were divided into the intentional and unintentional groups of sizes $N / 2$ each. From the simulations we evaluate, as a function of sample size and values of $\rho_{W, V}$, how wide the bounds are and their sampling variance.

Tables 2-4 show simulation results for $\rho_{W, V}, \beta_{2}^{N o}$, and $\beta_{2}^{\text {Yes }}$, respectively. The entries in the table show the sample size, $N$, the true value of the parameter in the simulation, and the simulation estimate of the mean and standard deviation of the sampling distribution of the estimated bounds. Also shown in Table 2 for $\rho_{W, V}$ are two numbers for each simulation case, $n_{p}$ and $n_{c}$. Though the population correlation matrix that simulated the data was positive definite, due to sampling variability the resulting estimate of this matrix in simulated data might not be positive definite. Thus $n_{p}$ is the number of sample correlation matrices out of 200 simulations that were positive definite. Also, of this number, the procedure outlined in Section 3.3 did not always produce a continuous interval for $\rho_{W, V}$. Often the sample correlation matrix was positive definite for values of $\rho_{W, V}$ near 1 , though the matrix was very near singular in these 
Table 4. Same Results as Described in Table 3 Except for the Parameter $\beta_{2}^{\text {Yes }}$

\begin{tabular}{|l|l|l|cc|cc|cc|cc|}
\hline \multicolumn{2}{|c}{$N$} & \multicolumn{2}{c|}{100} & \multicolumn{2}{c|}{500} & \multicolumn{2}{c|}{1000} & \multicolumn{2}{c|}{10000} \\
\hline$\rho_{W, V}$ & \multirow{2}{*}{$\beta_{2}^{\text {Yes }}$} & & $\min$ & $\max$ & $\min$ & $\max$ & $\min$ & $\max$ & $\min$ & $\max$ \\
\hline \multirow{2}{*}{0.3} & \multirow{2}{*}{-0.175} & mean & -0.212 & 0.580 & -0.183 & 0.796 & -0.207 & 0.862 & -0.216 & 1.088 \\
\cline { 3 - 11 } & & $\mathrm{sd}$ & 0.256 & 0.525 & 0.065 & 0.412 & 0.049 & 0.367 & 0.016 & 0.096 \\
\hline \multirow{2}{*}{0.4} & \multirow{2}{*}{0.108} & mean & -0.233 & 0.555 & -0.194 & 0.850 & -0.201 & 0.864 & -0.218 & 1.088 \\
\cline { 3 - 11 } & & sd & 0.294 & 0.625 & 0.063 & 0.428 & 0.049 & 0.375 & 0.017 & 0.074 \\
\hline \multirow{2}{*}{0.5} & \multirow{2}{*}{0.015} & mean & -0.250 & 0.810 & -0.197 & 0.848 & -0.201 & 0.860 & -0.215 & 1.085 \\
\cline { 3 - 11 } & & sd & 0.336 & 0.909 & 0.067 & 0.423 & 0.0510 & 0.396 & 0.018 & 0.079 \\
\hline \multirow{2}{*}{0.6} & \multirow{2}{*}{0.310} & mean & -0.222 & 0.749 & -0.199 & 0.842 & -0.206 & 0.886 & -0.216 & 1.095 \\
\cline { 3 - 10 } & & sd & 0.260 & 0.508 & 0.072 & 0.425 & 0.043 & 0.380 & 0.016 & 0.064 \\
\hline
\end{tabular}

cases. The number $n_{c}$ is the number of simulations where the estimated bounds contained a continuous interval, i.e., valid bounds. The mean and standard deviation of the sampling distributions for the lower and upper bounds was computed from the number $n_{c}$ in all Tables $2-4$.

The simulations provide insight into the sampling variability of the bounds at smaller values of $N$. The estimated means of the sampling distributions for the minimum and maximum contain the true parameter values. One exception are the bounds for $\rho_{W, V}$ when its true value is 0.6 and $N=100$. However, the sampling variability of all bounds is large when $N=100$. The bounds involve estimates of several population correlations and standard deviations. The sampling variability becomes smaller when $N$ is larger than 1000. This would suggest some caution in interpreting the accuracy of the bounds in the mice data example where $N$ was equal to 131 .

The sampling variability at smaller $N$ also causes some estimated correlation matrices from simulated data to not be positive definite. For example, when $N=100$, only around 150 out of 200 simulated data sets had correlation matrices that were positive definite. Also for some simulations at small values of $N$, some estimated bounds are not valid bounds, i.e., since $n_{p}>n_{c}$. These cases disappeared as $N$ became larger.

The width between the bounds does not depend on the true value of $\rho_{W, V}$ as would be expected since $\rho_{W, V}$ is not estimable in observed data. It should be noted that the parametric bootstrap simulation procedure simulated data using parameter estimates from the data example as model parameter values in the simulation model, thus the true value of $\rho_{W, V}$ was constrained to be between the bounds computed from the data. The width between the bounds also does not depend on sample size, $N$. Increasing $N$ only decreases the sampling variability of the bounds. The interval between the bounds will become narrower as one finds covariates that are highly predictive of the weight loss variables, $W$ and $V$.

\section{DISCUSSION}

A method was presented to estimate bounds for a nonestimable correlation which, in turn, were used to estimate bounds for a causal parameter in a linear model. Though valid bounds (bounds that contain a continuous interval) were obtained in the data example, the simulations suggest that the method will not always produce valid bounds when sample sizes are small due to sampling variability in the estimates, and the complicated relationship between $\rho_{Y^{(1)}, W^{(1)}}$ and $\rho_{W, V}$. For example, using the relationship between $\rho_{Y^{(1)}, W^{(1)}}$ and $\rho_{W, V}$ on the data example as outlined in Section 3.2, set of plausible values for $\rho_{W, V}$ was the union of the two intervals, $(0.069,0.676)$ and $(0.792$, 0.890). The upper range was excluded using the intersection defined in equation (17). In some simulations, however, this does not always happen and one is left with invalid bounds. Additional covariates could help resolve cases such as these. Let $U_{1}$ denote baseline weight and $U_{2}$ be a baseline weight (centered about the mean) by weight loss interaction. The bounds for $\rho_{W, V}$ based on the correlation matrix for $\left(W^{(1)}, V, U_{1}, U_{2}\right)^{\prime}$ are $(0.096,0.692)$ which does, in fact, exclude the upper range $(0.792,0.890)$ even without the use of equation (17).

In the data example, baseline weight was useful in tightening the bounds for $\rho_{W, V}$ because it was a good linear predictor of $W$ and of $V$. This presents the problem of collinearity when used in the causal model. Questions arise as to whether high baseline weight is associated with higher weight loss which then leads to decreased mortality, or whether high baseline weight itself is associated with mortality. Typically, the potential outcomes framework for a two treatment comparison would assume a potential outcome response variable corresponding to each possible treatment. We have assumed that assignment to intention was random and that there is no interference between subjects (Rubin, 1980), but we have two different potential outcome variables to this assignment, the pairs $(W, V)$ and $\left(Y^{(0)}, Y^{(1)}\right)$. This complicates the interpretation of effect of intention on the variables, $\left(Y^{(0)}, Y^{(1)}\right)$. Further challenges are encountered if relaxing the random assignment assumption and dealing with the more practical scenario, at least with human studies, of nonrandom assignment.

Some results from other applications have been reported by others that might be helpful in addressing some issues 
encountered in this application. One possibility is to borrow from the concepts related to partial compliance in clinical trials (Jin and Rubin, 2008). In the application considered here, weight loss might be thought of as "dosage received" after the assignment of intention (i.e., treatment). Material on principal stratification (Frangakis and Rubin, 2002) might then help to more clearly define the relationship between "intention to lose weight" and some subsequent measure of health or mortality. Graphical models or path analyses (see Pearl, 2000, for a reference) might also be useful.

The causal models considered here are rather simplistic and do not incorporate many of the potential confounders and nonlinearities in the relationship between weight loss and mortality that have been seen or suspected in other studies (Allison et al., 1997; Fontaine and Allison, 2001; Brock et al., 2006). More complicated models could be considered via Bayesian MCMC techniques that can accommodate censoring, nonlinear effects, and do not necessarily require that the slope parameter on UWL, $\beta_{1}$, be the same in both groups. At issue still is the choice of a prior for nonestimable parameters. The methods described here could suggest priors that are more informative by ruling out implausible values for $\rho_{W, V}$. This work is in progress (Yi et al., 2008).

A difficulty in human data is to find some variable that is predictive of subsequent weight loss (Allison and Engel, 1995). Unfortunately "intention to lose" has been shown to be a weak predictor, at best, of subsequent weight loss, especially among older persons who have the highest death rates (Lee et al., 2004), because subsequent weight loss seems to be confounded by many other possible variables (Hardy and Kuh, 2006). Other study designs might prove useful in adjusting for potential confounders thus making the effect of intention on subsequent weight loss more clear. Sorensen et al. (2005) reported results from an 18 year mortality study of overweight individuals that was based on The Finnish Twin Cohort. They did not specifically analyze their data using a matched-pairs type of analysis since many of the twin pairs lost their co-twin during the screening process. Matching can have appeal when drawing causal inferences using observational data (Rosenbaum and Rubin, 1983) and when bounding nonestimable parameters in randomized experiments (Gadbury et al., 2004; Albert et al., 2006).

Our treatment of this topic can be seen as an exemplar of a broader class of approaches all aimed at reducing the influence of putative confounding biases in assessing the relation between aspects of obesity and mortality rate. Such putative biases include the so-called 'late-life bias' 'reverse-causation bias,' and 'regression-dilution bias' (cf., Greenberg, 2001, 2002). Future research should address those additional hypothesized biases and see if our method could be combined with methods aimed at reducing those hypothesized biases.

In our study, we had the advantage of having well measured weights. In many human studies, weights are selfreported and this could conceivably introduce additional biases. Such conceivable biases also merit further investigation. Finally, one should note that any conclusions from a sample from one population must be generalized to other populations with caution. Hence it will be useful to conduct such analyses across multiple species and multiple human populations to assess the consistency of results. For additional discussion of these issues see Campbell and Kenny (1999).

\section{ACKNOWLEDGMENTS}

The project described was supported by Grant Number R01DK067487 from the National Institute Of Diabetes And Digestive And Kidney Diseases. The content is solely the responsibility of the authors and does not necessarily represent the official views of the National Institute Of Diabetes And Digestive And Kidney Diseases or the National Institutes of Health. The authors thank Richard Weindruch for use of the data.

\section{APPENDIX A}

An alternative derivation of equation (9) using partial regression coefficients is shown here. In particular, a functional relationship between two nonestimable correlation parameters, $\rho_{W, V}$ and $\rho_{Y^{(1)}, W^{(1)}}$, is established. Equation (5) gives $\beta_{1}=\frac{\operatorname{Cov}\left(Y^{(0)}, W^{(0)}\right)}{\operatorname{Var}(W)}$.

Equation (8) gives two forms of $\beta_{2}$ which are

$$
\begin{aligned}
& \beta_{2}^{*}=\frac{\operatorname{Cov}\left(Y^{(1)}, V \mid W^{(1)}\right)}{\operatorname{Var}\left(V \mid W^{(1)}\right)}, \\
& \beta_{1}-\beta_{2}^{* *}=\frac{\operatorname{Cov}\left(Y^{(1)}, W^{(1)} \mid V\right)}{\operatorname{Var}\left(W^{(1)} \mid V\right)} \text { or } \\
& \beta_{2}^{* *}=\beta_{1}-\frac{\operatorname{Cov}\left(Y^{(1)}, W^{(1)} \mid V\right)}{\operatorname{Var}\left(W^{(1)} \mid V\right)} .
\end{aligned}
$$

Using the following identity,

$$
\begin{aligned}
\operatorname{Var} & \left(Y^{(1)}, V \mid W^{(1)}\right) \\
& =\left[\begin{array}{cc}
\sigma_{Y^{(1)}}^{2} & \sigma_{Y^{(1)}, V} \\
\sigma_{Y^{(1)}, V} & \sigma_{V}^{2}
\end{array}\right] \\
& -\frac{1}{\sigma_{W}^{2}}\left[\begin{array}{cc}
\sigma_{Y^{(1)}, W^{(1)}}^{2} & \sigma_{Y^{(1)}, W^{(1)}} \sigma_{W, V} \\
\sigma_{Y^{(1)}, W^{(1)}} \sigma_{W, V} & \sigma_{W, V}^{2}
\end{array}\right]
\end{aligned}
$$

we get $\operatorname{Cov}\left(Y^{(1)}, V \mid W^{(1)}\right)=\sigma_{Y^{(1)}} \sigma_{V}\left[\rho_{Y^{(1)}, V}-\rho_{Y^{(1)}, W^{(1)}} \times\right.$ $\left.\rho_{W, V}\right]$, and $\operatorname{Var}\left(V \mid W^{(1)}\right)=\sigma_{V}^{2}\left(1-\rho_{W, V}^{2}\right)$, so,

$$
\beta_{2}^{*}=\frac{\sigma_{Y^{(1)}}\left[\rho_{Y^{(1)}, V}-\rho_{Y^{(1)}, W^{(1)}} \rho_{W, V}\right]}{\sigma_{V}\left(1-\rho_{W, V}^{2}\right)} .
$$

Similarly, it can be shown that,

$$
\begin{aligned}
\beta_{2}^{* *}= & \frac{1}{\sigma_{W}}\left[\sigma_{Y^{(0)}} \rho_{Y^{(0)}, W^{(0)}}\right. \\
& \left.-\frac{\sigma_{Y^{(1)}}\left[\rho_{Y^{(1)}, W^{(1)}}-\rho_{Y^{(1)}, V} \rho_{W, V}\right]}{\left(1-\rho_{W, V}^{2}\right)}\right] .
\end{aligned}
$$


Rearranging equation (A1) we obtain, $\rho_{Y^{(1)}, W^{(1)}}=\frac{\rho_{Y^{(1)}, V}}{\rho_{W, V}}-$ $\frac{\beta_{2}^{*} \sigma_{V}\left(1-\rho_{W, V}^{2}\right)}{\sigma_{Y^{(1)}} \rho_{W, V}}$. It is this equation combined with $\beta_{2}^{*}=\beta_{2}^{* *}=$ $\beta_{2}$ that gives the functional relationship between $\rho_{Y^{(1)}, W^{(1)}}$ and $\rho_{W, V}$ shown in equation (10). Continuing the derivation, a substitution back into equation (A2) of $\beta_{2}^{* *}$ and using $\beta_{2}^{*}=$ $\beta_{2}^{* *}=\beta_{2}$ gives,

$$
\begin{aligned}
\beta_{2}= & \frac{1}{\sigma_{W}}\left[\sigma_{Y^{(0)}} \rho_{Y^{(0)}, W^{(0)}}\right. \\
- & \left.\frac{\sigma_{Y^{(1)}}\left[\frac{\rho_{Y^{(1)}, V}}{\rho_{W, V}}-\frac{\beta_{2} \sigma_{V}\left(1-\rho_{W, V}^{2}\right)}{\sigma_{Y^{(1)}} \rho_{W, V}}-\rho_{Y^{(1)}, V} \rho_{W, V}\right]}{\left(1-\rho_{W, V}^{2}\right)}\right] \\
\beta_{2}= & \frac{\sigma_{Y^{(0)}} \rho_{Y^{(0)}, W^{(0)}} \sigma_{Y^{(1)}} \rho_{W, V}\left(1-\rho_{W, V}^{2}\right)}{\sigma_{W} \sigma_{Y^{(1)}} \rho_{W, V}\left(1-\rho_{W, V}^{2}\right)} \\
& -\frac{\sigma_{Y^{(1)}}\left[\rho_{Y^{(1)}, V} \sigma_{Y^{(1)}}-\rho_{Y^{(1)}, V} \rho_{W, V} \sigma_{Y^{(1)}} \rho_{W, V}\right]}{\sigma_{W} \sigma_{Y^{(1)}} \rho_{W, V}\left(1-\rho_{W, V}^{2}\right)} \\
& +\frac{\beta_{2} \sigma_{Y^{(1)}} \sigma_{V}\left(1-\rho_{W, V}^{2}\right)}{\sigma_{W} \sigma_{Y^{(1)}} \rho_{W, V}\left(1-\rho_{W, V}^{2}\right)}
\end{aligned}
$$

which after some algebra yields,

$$
\beta_{2}=\frac{\sigma_{Y^{(1)}} \rho_{Y^{(1)}, V}-\sigma_{Y^{(0)}} \rho_{Y^{(0)}, W^{(0)}} \rho_{W, V}}{\sigma_{V}-\sigma_{W} \rho_{W, V}}
$$

as given in equation (9).

\section{APPENDIX B. WITH A COVARIATE}

Equating $\beta_{2}^{*}=\beta_{2}^{* *}$ that were given in Section 3.2, an equation of $\rho_{Y^{(1)}, W^{(1)}}$ as a function of $\rho_{W, V}$ can be obtained and this function substituted back to either one of the $\beta_{2}^{*}$ or $\beta_{2}^{* *}$.

Define

$$
\begin{aligned}
& E_{1}=\rho_{Y^{(1)}, V}\left(1-\rho_{W, U}^{2}\right)+\rho_{Y(1), U}\left(\rho_{W, V} \rho_{W, U}-\rho_{V, U}\right) \\
& D=1-\rho_{W, U}^{2}-\rho_{V, U}^{2}-\rho_{W, V}^{2}+2 \rho_{W, V} \rho_{W, U} \rho_{V, U}, \\
& E_{2}=\rho_{Y^{(1)}, V}\left(\rho_{W, U} \rho_{V, U}-\rho_{W, V}\right) \\
& \quad+\rho_{Y^{(1)}, U}\left(\rho_{W, V} \rho_{V, U}-\rho_{W, U}\right) .
\end{aligned}
$$

Then,

$$
\begin{aligned}
& \beta_{2}^{*}= \frac{\sigma_{Y^{(1)}}}{\sigma_{V}}\left[\frac{\rho_{Y^{1}, W^{(1)}}\left(\rho_{W, U} \rho_{V, U}-\rho_{W, V}\right)+E_{1}}{D}\right], \\
& \beta_{2}^{* *}=\beta_{1}-\frac{\sigma_{Y^{(1)}}}{\sigma_{W}}\left[\frac{\rho_{Y^{(1)}, W^{(1)}}\left(1-\rho_{V, U}^{2}\right)+E_{2}}{D}\right], \quad \text { and } \\
& \rho_{Y^{(1)}, W^{(1)}=}\left[\frac{\sigma_{Y^{(1)}}\left(\rho_{W, U} \rho_{V, U}-\rho_{W, V}\right)}{\sigma_{V} D}+\frac{\sigma_{Y^{(1)}}\left(1-\rho_{V, U}^{2}\right)}{\sigma_{W} D}\right]^{-1} \\
& \times\left[\beta_{1}-\frac{\sigma_{Y^{(1)}} E_{2}}{\sigma_{W} D}-\frac{\sigma_{Y^{(1)}} E_{1}}{\sigma_{V} D}\right] .
\end{aligned}
$$

$$
\begin{aligned}
& A=\frac{\sigma_{Y^{(1)}}\left(\rho_{W, U} \rho_{V, U}-\rho_{W, V}\right)}{\sigma_{V} D}+\frac{\sigma_{Y^{(1)}}\left(1-\rho_{V, U}^{2}\right)}{\sigma_{W} D} \text { and } \\
& E_{12}=-\frac{\sigma_{Y^{(1)}}}{D}\left[\frac{E_{2}}{\sigma_{W}}+\frac{E_{1}}{\sigma_{V}}\right], \text { then } \\
& \rho_{Y^{(1)}, W^{(1)}}=\frac{\beta_{1}+E_{12}}{A} .
\end{aligned}
$$

Substituting equation (B1) back to $\beta_{2}^{*}$ and $\beta_{2}^{* *}$ gives

$$
\begin{aligned}
& \beta_{2}^{*}=\frac{\sigma_{Y^{(1)}}}{\sigma_{V} D}\left[\left(\frac{\beta_{1}+E_{12}}{A}\right)\left(\rho_{W, U} \rho_{V, U}-\rho_{W, V}\right)+E_{1}\right], \quad \text { and } \\
& \beta_{2}^{* *}=\beta_{1}-\frac{\sigma_{Y^{(1)}}}{\sigma_{W} D}\left[\left(\frac{\beta_{1}+E_{12}}{A}\right)\left(1-\rho_{V, U}^{2}\right)+E_{2}\right],
\end{aligned}
$$

respectively, and these two forms are algebraically equivalent.

\section{Received 18 March 2008}

\section{REFERENCES}

Albert, J. M., Gadbury, G. L., and Mascha, E. J. (2005). Assessing Treatment Effect Heterogeneity in Clinical Trials with Blocked Binary Outcomes. Biometrical Journal 47 662-673. MR2209062

Allison, D. B., and Engel, C. (1995). If We Live in a Deterministic World, Why Can't We Predict Treatment Outcome? Allison, D. B., and Pi-Sunyer, F. X. (eds.), Obesity Treatment: Establishing Goals, Improving Outcomes, and Reviewing the Research Agenda. Proceedings of a NATO Advanced Research Workshop. Plenum Press, New York.

Allison, D. B., Faith, M. S., Heo, M., and Kotler, D. P. (1997). A Hypothesis Concerning the U-shaped Relationship Between BMI and Mortality. American Journal of Epidemiology 146 339-349.

Allison, D. B., Zannolli, R., Faith, M. S., Heo, M., Pietrobelli, A., Van Itallie, T. B., Pi-Sunyer, F. X., and Heymsfield, S. B. (1999). Weight Loss Increases and Fat Loss Decreases All-Cause Mortality Rate: Results from Two Independent Cohort Studies. International Journal of Obesity 23 603-611.

Berkane, M., (ed.) (1997). Latent Variable Modeling and Applications to Causality. Springer, New York. MR1601263

Bollen, K. A. (2002). Latent Variables in Psychology and the Social Sciences. Annu. Rev. Psychol. 53 605-634.

Brock, D. W., Keith, S. W., Elobeid, M. A., and Allison, D. B. (2007). Does Intentional Weight Loss Influence Mortality and Other Hard End Points Favorably? Confessions of a Closet Bayesian and Occam-ite. Proceedings of the 2006 International Congress on Obesity. [CD-ROM], Sydney Australia, Sep. 3-8, 2006. Paper \# ISO111.

Campbell, D. T., and Kenny, D. A. (1999). A Primer on Regression Artifacts. The Guilford Press, New York, NY.

Coffey, C. S., Gadbury, G. L., Fontaine, K. R., Wang, C., Weindruch, R., and Allison, D. B. (2005). The Effect of Intentional Weight Loss as a Latent Variable Problem. Statistics in Medicine 24 941-954. MR2134548

Efron, B., and Tibshirani, R. J. (1993). An Introduction to the Bootstrap, Chapman \& Hall, New York. MR1270903

Fontaine, K. R., and Allison, D. B. (2001). Does Intentional Weight Loss Affect Mortality Rate? Eating Behaviors: An International Journal 2 87-95.

Frangakis, C. E., and Rubin, D. B. (2002). Principal Stratification in Causal Inference. Biometrics 58 21-29. MR1891039 
Gadbury, G. L., and Iyer, H. K. (2000). Unit-Treatment Interaction and its Practical Consequences. Biometrics 56 882-885.

Gadbury, G. L, Iyer, H. K., and Albert, J. M. (2004). Individual Treatment Effects in Randomized Trials with Binary Outcomes. Journal of Statistical Planning and Inference 121 163-174. MR2038814

Graybill, F. A. (1976). Theory and Application of the Linear Model. Duxbury, Pacific Grove, California. MR0455218

Gregg, E. W., Gerzoff, R. B., Thompson, T. J., and Williamson, D. F. (2003). Intentional Weight Loss and Death in Overweight and Obese U.S. Adults 35 Years of Age and Older, Annals of Internal Medicine 138(5) 383-389.

Gregg, E. W., Gerzoff, R. B., Thompson, T. J., and Williamson, D. F. (2004). Trying to Lose Weight, Losing Weight, and 9-year Mortality in Overweight U.S. Adults with Diabetes, Diabetes Care 27 657-662.

Greenberg, J. A. (2001). Biases in the mortality risk versus body mass index relationship in the NHANES-1 Epidemiologic Followup study. Int. J. Obes. Relat. Metab. Disord. 25 1071-1078.

Greenberg, J. A. (2002). Hypothesis - The J-shaped follow-up relation between mortality risk and disease risk-factor is due to statistical confounding. Medical Hypotheses 59 568-576.

Hardy, R., and Kuh, D. (2006). Commentary: BMI and Mortality in the Elderly - a Life Course Perspective. International Journal of Epidemiology 35 179-180.

Heckman, J. J., and Vytlach, E. J. (1999). Local Instrumental Variables and Latent Variable Models for Identifying and Bounding Treatment Effects. Proceedings of the National. Academy of Science 96 4730-4734. MR1683940

Jin, H., and Rubin, D. B. (2008). Principal Stratification for Causal Inference with Extended Partial Compliance. Journal of the American Statistical Association 103 101-111.

Lee, J. S., Kritchevsky, S. B., Tylavsky, F. A., Harris, T., Everhart, J., Simonsick, E. M., Rubin, S. M., and Newman, A. B. (2004). Health, Aging and Body Composition (Health ABC) Study. Weight-loss Intention in the Well-Functioning, Community-Dwelling Elderly: Associations with Diet Quality, Physical Activity, and Weight Change. American Journal of Clinical Nutrition 80 466-474.

Pearl, J. (2000). Causality. Cambridge University Press, New York. MR1744773

Rosenbaum, P. R. (1991). Discussing hidden bias in observational studies. Annals of Internal Medicine 115 901-905.

Rosenbaum, P. R. (1995). Observational Studies. Springer-Verlag, New York. MR1353914

Rosenbaum, P. R., and Rubin, D. B. (1983). The Central Role of the Propensity Score in Observational Studies for Causal Effects. Biometrika 70 41-55. MR0742974

Rubin, D. B. (1974). Estimating Causal Effects of Treatments in Randomized and Nonrandomized Studies. Journal of Educational Psychology 66 688-701.

Rubin, D. B. (1980). Comment on 'Randomization Analysis of Experimental Data: The Fisher Randomization Test,' by D. Basu. Journal of the American Statistical Association 75 591-593. MR0590687

Shipley, B. (2000). Cause and Correlation in Biology. Cambridge University Press, Cambridge, UK.

Sorensen, T. I. (2003). Weight loss Causes Increased Mortality: Pros. Obesity Reviews 4 3-7.

Sorensen, T. I., Rissanen, R., Korkeila, M., and Kaprio, J. (2005) Intention to Lose Weight, Weight Changes, and 18-year Mortality in Overweight Individuals Without Co-morbidities. PLoS Medicine $20510-0520$

Weinsier, R. L. (1987). Etiology, Complications, and Treatment of Obesity. A Clinician's Guide. The Alabama Journal of Medical Sciences 24 435-442.
Williamson, D. F. (1996). Lingering Questions About Intentional Weight Loss. Nutrition 12 819-820.

Yang, D., Fontaine, K. R., Wang, C., and Allison, D. B. (2003). Weight Loss Causes Increased Mortality: Cons. Obesity Reviews 4 9-16.

Yi, N., Ding, S., Keith, S. W., Coffey, C. S., and Allison, D. B. (2008). Bayesian Analysis of the Effect of Intentional Weight Loss on Mortality Rate. (Working paper).

\section{Gary L. Gadbury}

Department of Statistics

Kansas State University

Manhattan, KS 66506, USA

E-mail address: gadbury@ksu.edu

Department of Mathematics and Statistics

Missouri University of Science and Technology

Rolla, MO 65409, USA

and

Department of Biostatistics

School of Public Health

University of Alabama at Birmingham

Birmingham, AL, USA

Thidaporn Supapakorn

Department of Statistics

Kansas State University

Manhattan, KS 66506, USA

and

Department of Mathematics and Statistics

Missouri University of Science and Technology

Rolla, MO 65409, USA

Christopher S. Coffey

Department of Biostatistics

School of Public Health

University of Alabama at Birmingham

Birmingham, AL, USA

Scott W. Keith

Department of Biostatistics

School of Public Health

University of Alabama at Birmingham

Birmingham, AL, USA

David B. Allison

Department of Biostatistics

School of Public Health

University of Alabama at Birmingham

Birmingham, AL, USA

and

Clinical Nutrition Research Center

University of Alabama at Birmingham

Birmingham, AL, USA 